\title{
Centroids and derivations of low-dimensional Leibniz algebra
}

\begin{abstract}
In this paper we introduce the concept of centroid and derivation of Leibniz algebras. By using the classification results of Leibniz algebras obtained earlier, we describe the centroids and derivations of low-dimensional Leibniz algebras. We also study some properties of centroids of Leibniz algebras and use these properties to categorize the algebras to have socalled small centroids. The description of the derivations enables us to specify an important subclass of Leibniz algebras called characteristically nilpotent.
\end{abstract}

Keyword: Centroids; Leibniz algebra 